\title{
On steady shell formation in stellar atmospheres
}

\section{Energy balance in a non-polytropic stellar envelope}

\author{
A. Kakouris ${ }^{1,2, \star}$ \\ 1 Section of Astrophysics, Astronomy \& Mechanics, Physics Department, University of Athens Panepistimiopolis, \\ 15783 Zografos, Athens, Greece \\ 2 Hellenic Air Force Academy, Dekelia, Attiki, Greece
}

Received 25 October 2000 / Accepted 12 April 2001

\begin{abstract}
The energy balance of the analytical solutions of Kakouris \& Moussas (1997) for a steady state of an externally heated/cooled 2-D circumstellar envelope is investigated. It is found that the required heating/cooling rates are physically realistic and can be related to specific microscopic mechanisms. We find that in the subsonic region of the wind the fluid is mechanically heated. In the supersonic stellar envelope the fluid is cooled at a rate which is consistent with radiative cooling to space. The energy balance of steady shell or blob formation in the envelopes of luminous early or late type supergiants is also investigated (Kakouris \& Moussas 1998). We find that radiative cooling occurs in the intermediate deceleration region of the three-zone envelope. Indicative of the local thermodynamic processes is the effective polytropic index $\alpha$ which takes values close to the star between 1 and 4 , becoming $\simeq 2$ at larger distances. The heated subsonic region close to the stellar surface is isothermal and becomes adiabatic at the sonic transition. We find that the polytropic index $\alpha$ is less than unity in the vicinity of the dense blob, indicating that the region may be dominated by convection.
\end{abstract}

Key words. hydrodynamics - methods: analytical - stars: atmospheres - supergiants - stars: mass-loss

\section{Introduction}

Shell features are often observed in the spectra of early or late type stars, mainly giants, as well as by deep optical imaging obtained from HST (Hauck \& Jaschek 2000; Winters et al. 2000; Mauron \& Huggins 2000; Villada et al. 1999; Olofsson et al. 1998; Jura 1984; Zuckerman 1980). Shell formation in stellar atmospheres, or a shellphase during evolution, is a feature of the mass loss mechanism which can be addressed self-consistently by radiative hydrodynamics. Stellar winds emanating from early type supergiants with low terminal velocities may be caused by the thin-line-radiative driving described by Chen \& Marlborough (1994). By contrast, the strong winds observed in early main-sequence stars are traditionally modeled by the thick-line-driven wind theory (Castor et al. 1975) which is based on the Sobolev approximation. However, the reduced acceleration near the stellar surface and the low terminal velocity of winds from supergiants cannot be accounted for thick-line driving (as for example has been extensively discussed by Lamers (1986) for $P$ Cyg), as well as the possible deceleration regions in the stellar envelope (Kuan \& Kuhi 1975). Assuming

* e-mail: akakour@cc.uoa.gr an optically thin stellar atmosphere, Kakouris \& Moussas (1998), hereafter Paper I, proposed an alternative thermoradiative mechanism which can describe the deceleration region in the stellar envelope and steady shell formation under certain conditions. Similar ideas on three zone stellar envelopes were proposed earlier by Nugis et al. (1979).

In the present article we extend the work of Kakouris (1997) and Kakouris \& Moussas (1996, 1997, 1998) in which an analytical 2-D solution for thermo-radiatively driven stellar outflows was found. When the velocity field and the fluid density and pressure are given, the heating function is easily calculated and the feasibility of the predicted heating rates can be discussed. The present analysis provides an a posteriori test of the feasibility of this class of solutions.

This calculation is crucial for non-polytropic dynamical models because no a priori assumptions on energetics are made in these models (cf. Low \& Tsinganos 1986; Sauty \& Tsinganos 1994). This procedure provides an inverse method for modeling the envelope that can elucidate the poorly understood heating mechanisms (even for the solar wind, Totten et al. 1996). The present analytical calculations of energy balance provide a detailed estimation of the required total heating/cooling rates as a function of the distance from the star, when the outflow dynamics are 
given. Assuming LTE we can calculate an effective polytropic index, which can indicate the local thermodynamic processes in the stellar envelope, as in studies of the solar wind and the terrestrial magnetosphere (cf. Totten et al. 1995, 1996; Pudovkin et al. 1997).

The paper is organized as follows: in Sect. 2 the formulation of the energetic terms and thermodynamic indices are summarized and in Sect. 3 two illustrative applications are presented. The first concerns a non-radiative, thermally driven outflow. The second is a shell application using the prototype of LBV, $P$ Cyg, as the central object, similar to the one treated in Paper I. The origin of the heating/cooling mechanisms is also discussed. Finally, in Sect. 4 the new results are summarized and discussed.

\section{Energetics of the non-polytropic outflow}

A steady, inviscid outflow from a central gravitational object under the influence of the object's radiation pressure, obeys mass conservation

$$
\nabla \cdot(\rho \boldsymbol{V})=0,
$$

and the steady state momentum equation

$\rho[\boldsymbol{V} \cdot \nabla] \boldsymbol{V}=-\nabla p+\boldsymbol{F}_{\mathrm{rad}}-(1-\Gamma) \rho \frac{G M}{r^{2}} \widehat{r}$,

where $\boldsymbol{V}$ is the flow velocity, $\rho$, the density, $p$ the pressure, $\boldsymbol{F}_{\text {rad }}$ the radiative force, $G$ the gravitational constant and $M$ the mass of the central object. The last term on the right hand side of the momentum equation is the volumetric effective gravitational force which has been reduced by a factor $(1-\Gamma)$ due to Thomson scattering of photons by the fluid free electrons. The factor $\Gamma$ is the ratio of the luminosity of the central object, $L$, to the Eddington luminosity, $L_{\mathrm{E}}$. Axisymmetry $(\partial / \partial \phi=0)$ and helicoidal flow geometry $\left(V_{\theta}=0\right)$ imply an angular momentum conserving azimuthal velocity field $V_{\phi} \sim 1 / r$ (here $r, \theta, \phi$ are spherical coordinates). The remaining components of the flow field and the thermodynamic variables can then be determined from Eqs. (1), (2). The fluid will be also assumed to be ideal, satisfying the equation of state

$p=\frac{k_{\mathrm{B}}}{\mu} \rho T$,

where $T$ the fluid temperature, $k_{\mathrm{B}}$ the Boltzmann's constant and $\mu$ the mean molecular mass which is equal to the half proton mass $\left(\mu=m_{\mathrm{p}} / 2\right)$ for an ionized neutral plasma with equal proton and electron temperatures. The fluid number density is defined as $n=\rho / \mu$.

Since the flow is not adiabatic and the usual polytropic assumption $\left(p \propto \rho^{\alpha}\right)$ cannot be adopted, such models are called non-polytropic. The heating function is obtained from the first law of thermodynamics where the per unit mass amount of heat, $\mathrm{d} Q$, required for consistency is given by

$\mathrm{d} Q=c_{\mathrm{V}} \mathrm{d} T+p \mathrm{~d}(1 / \rho)$, where $c_{\mathrm{V}}$ the specific heat of constant volume. The rate of the specific input energy is $\sigma=\mathrm{d} Q / \mathrm{d} t$ (in $\mathrm{erg} \mathrm{gr}^{-1} \mathrm{~s}^{-1}$ ) is

$\sigma(r, \theta)=[\boldsymbol{V} \cdot \nabla]\left(\frac{p}{(\gamma-1) \rho}\right)+p[\boldsymbol{V} \cdot \nabla]\left(\frac{1}{\rho}\right)$.

The last equation can also be expressed in terms of the volumetric rate of thermal energy $q=\rho \sigma$ (in erg $\mathrm{cm}^{-3} \mathrm{~s}^{-1}$ )

$q(r, \theta)=\rho \boldsymbol{V} \cdot\left[\nabla h-\frac{\nabla p}{\rho}\right]$,

where $h$ is the fluid specific enthalpy. Equations (4)-(6) are equivalent and for positive $\sigma$ (or $q$ ) the fluid is heated while for negative $\sigma$ (or $q$ ) the fluid cools. For adiabatic flows $\sigma=q=0$ and the enthalpy is given by $h_{\mathrm{ad}}=\int \mathrm{d} p / \rho$. In the present non-polytropic analysis (cf. Sauty \& Tsinganos 1994) the streamlines lie on helices on a circular conical surface of semi-vertical angle $\theta$. The enthalpy variation along a streamline $(\theta=$ const.) starting from the stellar radius $R_{*}$ is

$h=h_{\mathrm{ad}}+h_{\mathrm{q}}=\int_{s_{*}}^{s} \frac{1}{\rho} \frac{\mathrm{d} p}{\mathrm{~d} r} \mathrm{~d} s+\int_{s_{*}}^{s} \frac{q}{\rho V_{\mathrm{r}}} \mathrm{d} s$,

where $s$ is the path length along a streamline and the second term $h_{\mathrm{q}}$ is the enthalpy change due to heating/cooling. As a result, the Bernoulli integral, which gives the total energy flux density $E$ (sum of kinetic, thermal, gravitational, radiative), is not conserved along a streamline. This integral can be decomposed into a conserved (adiabatic) term, $I_{\mathrm{B}}$, and into a heating term $h_{\mathrm{q}}$

$E=I_{\mathrm{B}}+h_{\mathrm{q}}$,

where

$I_{\mathrm{B}}=\frac{1}{2} V_{\mathrm{r}}^{2}+\frac{1}{2} V_{\phi}^{2}+h_{\mathrm{ad}}+\Phi_{\text {grav }}+\Phi_{\mathrm{rad}}$,

with $\Phi_{\text {grav }}=-(1-\Gamma) G M / r$ and $\Phi_{\text {rad }}$ the radiative potential, is conserved along a streamline.

An effective polytropic index, $\alpha(r, \theta)$, can be defined along a streamline

$\alpha(r, \theta)=\left(\frac{\partial \ln p}{\partial \ln \rho}\right)_{\theta=\text { const. }}$.

If the outflow is isentropic $\alpha=\gamma=c_{\mathrm{p}} / c_{\mathrm{V}}$ where $c_{\mathrm{p}}$ and $c_{\mathrm{v}}$ the fluid specific heats of constant pressure and volume respectively which for a monoatomic gas is $\gamma=5 / 3$. In a quasi-static polytropic process, a constant specific heat can be defined as $c=\mathrm{d} Q / \mathrm{d} T$ where $\mathrm{d} Q$ the amount of heat, giving

$\alpha=\frac{c_{\mathrm{p}}-c}{c_{\mathrm{V}}-c}$.

For isentropic expansion $c=0$ and $\alpha=\gamma$. The polytropic index is generally positive $(\alpha=0$ for isobaric, $\alpha=1$ for isothermal, $\alpha=\gamma=5 / 3$ for adiabatic and $\alpha \rightarrow \infty$ for isometric processes), although it can be extended to negative values (Viala \& Horedt 1974). The study of varying polytropes was first applied to stellar interiors 
(cf. Eddington 1938) and in many cases it is not found in the interval $1 \leq \alpha \leq \gamma$. However, we will not accept values of $a$ less than 1 . In such envelope regions the equivalent polytropic thermodynamic description is invalidated and a local detailed analysis is required using the hydrodynamic equations taking into account the thermal and hydrodynamic timescales (Ryu \& Goodman 1992; Totten et al. 1995; Pudovkin et al. 1997).

\section{Applications}

If the radiative force in Eq. (2) is neglected (non-radiative case) or a thin-line radiative force of the form (14) is assumed, the steady state velocity components are determined to be

$V_{\mathrm{r}}=V_{\mathrm{p}} \sqrt{f_{1}(r)-f_{2}(r) \sin ^{2 \lambda} \theta}, V_{\phi}=V_{\mathrm{p}} \frac{R_{*}}{r} \omega \sin ^{\lambda} \theta$,

where $V_{\mathrm{p}}=\sqrt{2 k_{\mathrm{B}} T_{\mathrm{p}} / m_{\mathrm{p}}}$ and $T_{\mathrm{p}}$ a constant, $\omega$ is the dimensionless rotational velocity at the equatorial stellar surface and $\lambda$ a positive parameter of differential rotation. The analytic expression of $f_{1}, f_{2}$ is given in Kakouris \& Moussas (1997). This class of solutions has a radial velocity maximum at the equator $(\theta=\pi)$. The sonic surface where the outflow radial velocity becomes equal to the adiabatic sound speed $(c=\sqrt{\gamma p / \rho})$, is spherical and close to the stellar surface. The radial mass flux density (Eq. (1)) does not depend on co-latitude $\theta$ and varies as $r^{-2}$

$\rho(r, \theta)=\rho_{\mathrm{o}} \frac{R_{*}^{2} V_{\mathrm{p}}}{r^{2} V_{\mathrm{r}}(r, \theta)}$,

and the mass loss rate is $\dot{M}=4 \pi R_{*}^{2} \rho_{\mathrm{o}} V_{\mathrm{p}}$.

\subsection{Energy balance in the non-radiative case}

The central object in this application is a star with $M=$ $12 M_{\odot}$ and very low surface gravity $\log \left(g / g_{\odot}\right)=-4.1$, rotating with $V_{\text {rot }}=25 \mathrm{~km} \mathrm{~s}^{-1}$. The luminosity is $L_{*}=$ $3.8 \times 10^{4} L_{\odot}$. These parameters correspond to a K5I supergiant and the example is the same with the one presented in Kakouris \& Moussas (1997). The model parameters are chosen to be $\left(T_{\mathrm{p}}=10^{3}, A=800, \lambda=50\right)$. This example is a model of a "coronal wind" outflow (cf. Lamers \& Cassinelli 1999, Chapt. 5) with a maximum temperature at the stellar surface of $2 \times 10^{5} \mathrm{~K}$. We set the parameter $\rho_{\mathrm{o}}=10^{8}$ part. $\mathrm{cm}^{-3}$ which gives a mass loss rate of $10^{-8} M_{\odot} / \mathrm{yr}$. This example may be extended to be a model of outflows from cool supergiants if we include the radiation pressure force on dust grains after $\sim 3$ stellar radii from the giant where the temperature drops below $10^{3} \mathrm{~K}$ (Fig. 3). Note that the inclusion of dust will affect only the outer supersonic regions of the wind by increasing the terminal velocity, but both the mass loss rate and the wind structure near the star are unaffected.

The radial velocities (polar and equatorial) and the equatorial force balance are shown in Fig. 1. The flow is initially accelerated, becomes supersonic (sonic transition
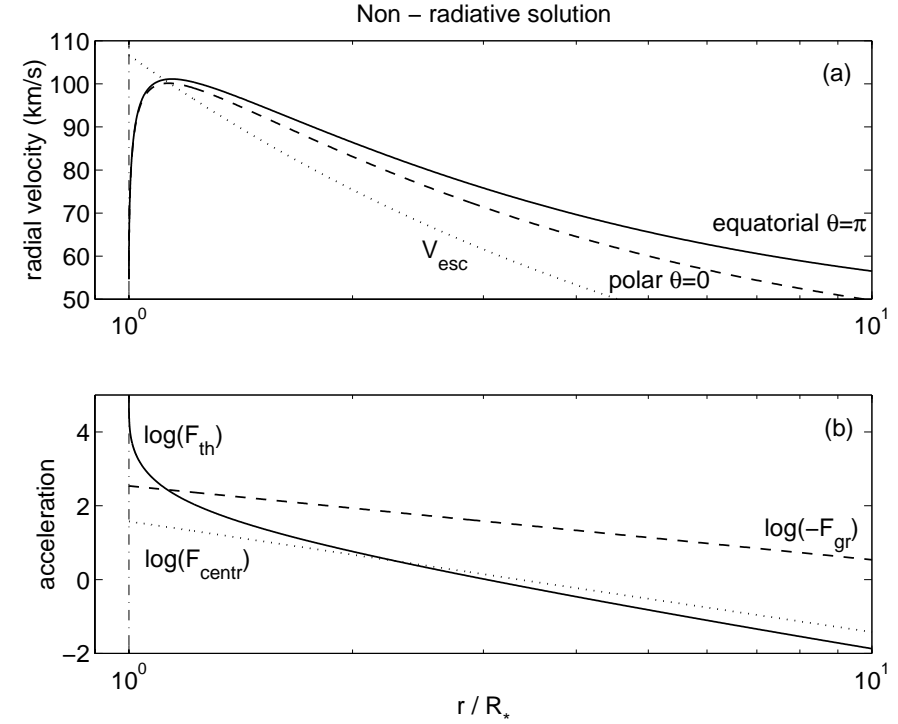

Fig. 1. a) Radial velocities and b) equatorial force balance for the non-radiative case. The solid curve in plot $\mathbf{a}$ ) is equatorial and the dashed is polar. The dotted curve shows the local escape speed. In the lower panel, the solid curve is the thermal pressure acceleration $(-(1 / \rho) \nabla p)$, the dashed is the gravitational $G M_{*} / r^{2}$ and the dotted is the centrifugal $V_{\phi}^{2} / r$. Accelerations are in $V_{\mathrm{p}}^{2} / R_{*} \simeq 5.9 \times 10^{-8} \mathrm{~km} / \mathrm{s}^{2}$ units. The parameters of the solution are given in Sect. 3.1.

at $1.0014 R_{*}$ ), and then exceeds the local escape speed. For larger distances the flow decelerates. The equatorial specific thermal energy rate $\sigma$ and the volumetric $q$ are shown in Fig. 2. Heating is needed only in the subsonic region. In the supersonic region the fluid cools. The respective equatorial effective polytropic index is shown in Fig. 3. The radial structure of the number density and temperature are also shown in the same plot. The subsonic region is isothermal close to the stellar surface and becomes gradually adiabatic at the sonic distance. In the supersonic cooling region the polytropic index exceeds $\gamma$, maximizes $(\sim 4)$ after the sonic radius and approaches 2 asymptotically. The polytropic index is acceptable and close to the common interval $[1, \gamma]$ despite the lack of initial assumptions on thermodynamic processes.

The volumetric heating/cooling rate depends on the mass loss rate, $\dot{M}$, which was chosen for the cooling rate to match with the estimates of Schmutzler \& Tscharnuter (1993) for the effective radiative cooling in optically thin plasmas.

\subsubsection{The heated inner envelope}

In the subsonic region, the solution indicates that the fluid is mechanically heated. The effective heat luminosity $\mathcal{L}_{\mathrm{h}}$ is found by integrating the heating volumetric rate over the subsonic region

$\mathcal{L}_{\mathrm{h}}=\int q \mathrm{~d} V=\int_{R_{*}}^{R_{\mathrm{s}}} q 4 \pi r^{2} \mathrm{~d} r$. 


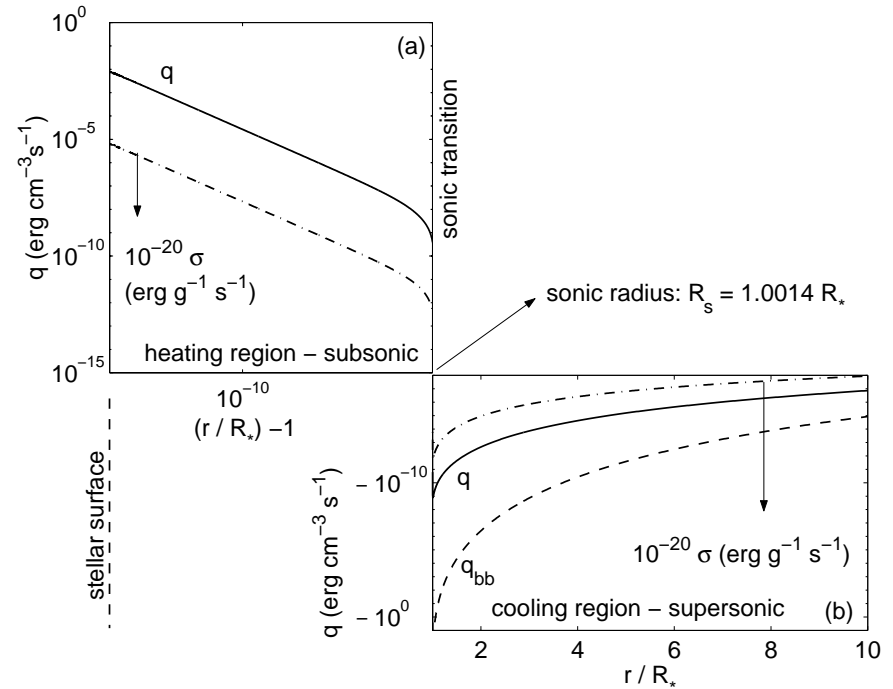

Fig. 2. Equatorial heating function for the non-radiative case. The solid curve shows the volumetric rate of heating $q$ (erg $\mathrm{cm}^{-3} \mathrm{~s}^{-1}$ ). The dash-dotted curve is the heating specific energy rate $\sigma\left(\mathrm{erg} \mathrm{g}^{-1} \mathrm{~s}^{-1}\right)$ multiplied by $10^{-20}$ to fit in scale. The stellar envelope is separated into the subsonic region (plot a)) where heating occurs and into the supersonic (plot b)) where the fluid cools. The sonic transition occurs close to the stellar surface $r_{\mathrm{s}}=1.0014 R_{*}$ and the subsonic region is resolved with the use of the variable $x=r / R_{*}-1$. The dashed curve in the supersonic region shows the local black-body volumetric emission rate $q_{\mathrm{bb}}\left(\mathrm{erg} \mathrm{cm}^{-3} \mathrm{~s}^{-1}\right)$ which is much greater than the thermal losses $q$.

In this example, the volumetric heating rate at the stellar surface, $q_{0}$, the incident mechanical heating flux $F_{0}$, and the heating luminosity, $\mathcal{L}_{\mathrm{h}}$, scale with $\dot{M}$ as

$q_{0}\left(\mathrm{erg} \mathrm{cm} \mathrm{cm}^{-3} \mathrm{~s}^{-1}\right) \simeq 1.5 \times 10^{7} \dot{M}\left(M_{\odot} / \mathrm{yr}\right)$

$F_{0}\left(\mathrm{erg} \mathrm{cm} \mathrm{cm}^{-2} \mathrm{~s}^{-1}\right) \simeq 2.5 \times 10^{10} \dot{M}\left(M_{\odot} / \mathrm{yr}\right)$,

$\mathcal{L}_{\mathrm{h}} / L_{*} \simeq 1.8 \dot{M}\left(M_{\odot} / \mathrm{yr}\right)$.

The function $q$ is shown in Figs. 4a,b for several mass loss rates which cover the range from low $10^{-14} M_{\odot} / \mathrm{yr}$ (solar case) to high $10^{-4} M_{\odot} / \mathrm{yr}$ (cool supergiants). As expected, the heating/cooling increases with $\dot{M}$. The chosen value for application is shown by the dashed curves $\left(\dot{M}=10^{-8} M_{\odot} / \mathrm{yr}\right)$, giving $\mathcal{L}_{\mathrm{h}} \simeq 1.8 \times 10^{-8} L_{*}$ and $F_{0} \simeq 0.27 \times 10^{3} \mathrm{erg} \mathrm{cm}^{-2} \mathrm{~s}^{-1}$. The heating flux $F$ as a function of the height above the stellar photosphere, $H$, for several $\dot{M}$ is shown in Fig. 4c. The mechanical heating decreases with radial distance from the photosphere in the subsonic region and the equivalent damping length for $q$ or $F, l_{\mathrm{q}}=-H / \log \left(q / q_{0}\right)$ or $l_{\mathrm{F}}=-H / \log \left(F / F_{0}\right)$, is shown in Fig. 4d. The flux damping length scales with height as $l_{\mathrm{F}}(\mathrm{km}) \simeq 1 / 3 \times 10^{3} \sqrt{H / \mathrm{km}}$.

The radiative losses (cooling) of the subsonic region, considered optically thin at a constant temperature of $2 \times 10^{5} \mathrm{~K}$ and density $7.4 \times 10^{6} \mathrm{~cm}^{-3}$, is of the order of $10^{-8} \mathrm{erg} \mathrm{cm}^{-3} \mathrm{~s}^{-1}$ and compared to the heating rate, $q$, they can be neglected.

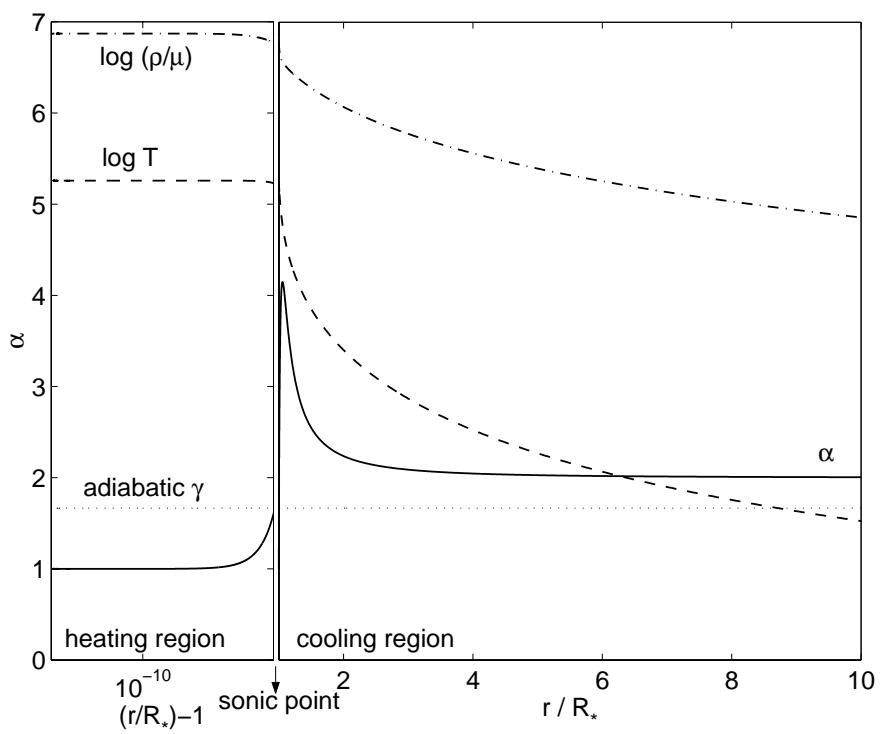

Fig. 3. Equatorial effective polytropic index $\alpha$ (solid line), temperature (dashed line) and number density (dash-dotted line) for the non-radiative case. The adiabatic index $\gamma=5 / 3$ is also shown by the dotted line.
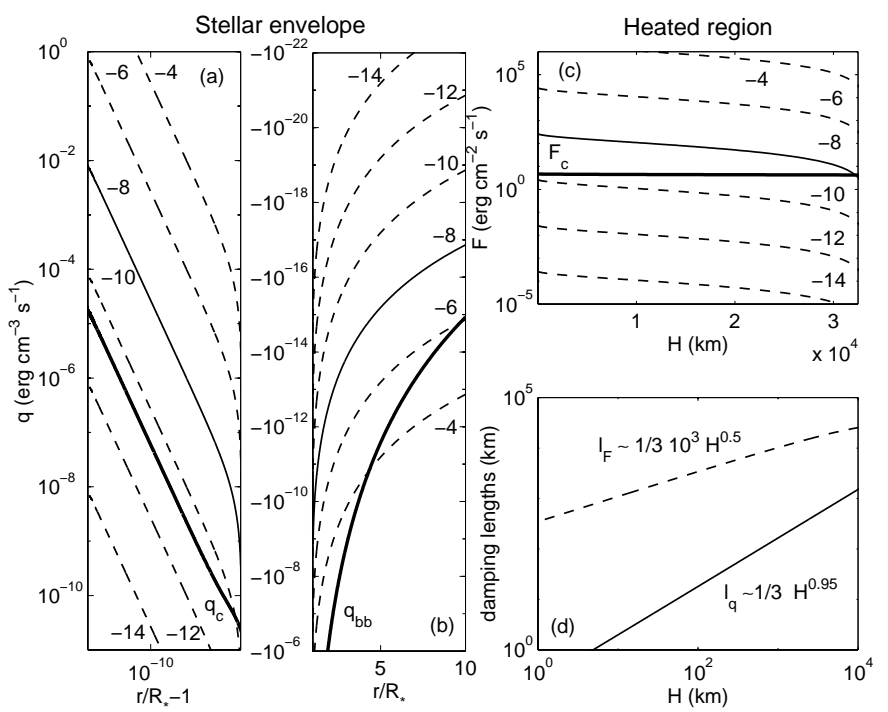

Fig. 4. Equatorial heating/cooling functions of the nonradiative case for several mass loss rates. a) volumetric heating rate, b) volumetric cooling rate, c) mechanical heating flux as a function of the height above the stellar photosphere, $H, \mathbf{d}$ ) damping lengths of the volumetric heating rate, $l_{\mathrm{q}}$, and of the heating flux, $l_{\mathrm{F}}$ as functions of $H$. The numbers close to the dashed curves in plots a-c) indicate the $\log \left(\dot{M} /\left(M_{\odot} \mathrm{yr}^{-1}\right)\right)$. The solid lines in the same plots indicate the applied case in Sect. 3.1. Note that the solid bold lines in plots a) and c) show the conductive heating. The bold solid line in plot b) shows the black-body emission rate.

The heat transfer rate by conduction is $q_{\mathrm{c}}=$ $-\nabla \cdot\left(\kappa_{\mathrm{c}} \nabla T\right)$ where $\kappa_{\mathrm{c}}$ is the thermal conductivity of the fluid. Ulmschneider's $(1970,1977)$ calculation for the coefficient of molecular thermal conductivity of a hydrogen gas is for $T>10^{4} \mathrm{~K}, \kappa_{\mathrm{c}}=10^{-6} T^{5 / 2} \mathrm{erg} \mathrm{cm}^{-1} \mathrm{~s}^{-1}$ and $\kappa_{\mathrm{c}}=35 \mathrm{Terg} \mathrm{cm}^{-1} \mathrm{~s}^{-1}$ for $T<5 \times 10^{3} \mathrm{~K}$. The conductive 

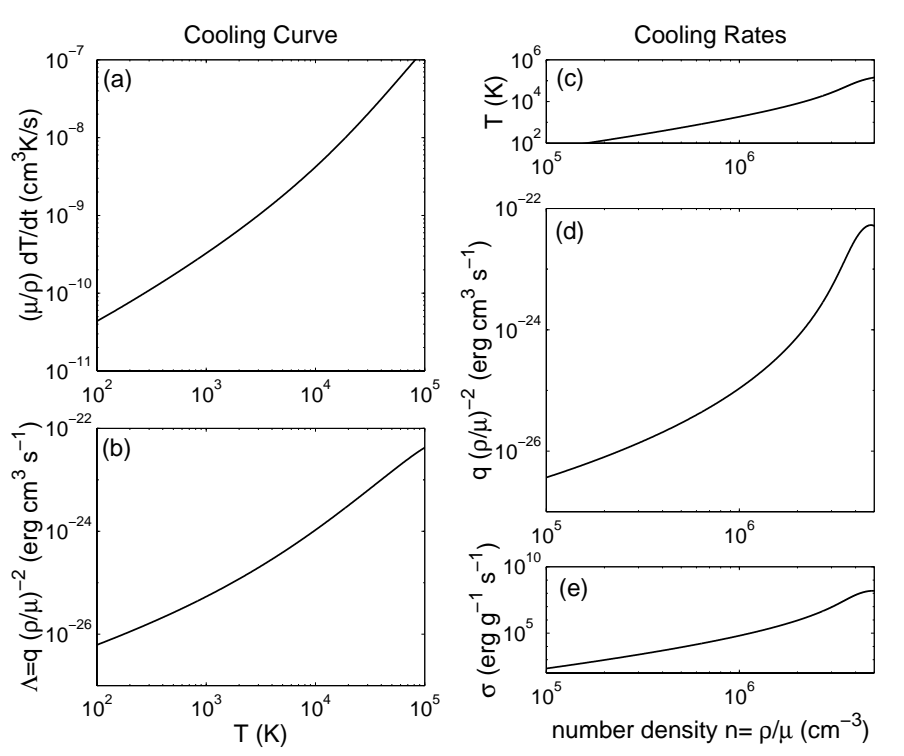

Fig. 5. Equatorial cooling curve (plot a)) and cooling rates for the non-radiative case. $n=\rho / \mu$ is the number density. The cooling rate $\Lambda=q n^{-2}\left(\mathrm{erg} \mathrm{cm}^{3} \mathrm{~s}^{-1}\right)$ is shown as a function of temperature (plot $\mathbf{b})$ ) or of the number density (plot $\mathbf{d})$ ). The cooling specific energy rate $\sigma$ is shown as function of $n$ (plot $\mathbf{e}))$ as well as the temperature versus $n$ (plot $\mathbf{c})$ ).

heat rate, $q_{\mathrm{c}}$, and the conductive heat flux $F_{\mathrm{c}}$ for the temperature of Fig. 3, are shown in Figs. 4a,c. Note that although the conductive heat rate, $q_{\mathrm{c}}$, exhibits similar radial dependence with $q$, it is three orders of magnitude smaller than the required heating. This heating estimate could be amended if we consider that the stellar photosphere is turbulent (cf. Cally 1990). But in any case laminar conductive heat transfer could be considered responsible for the heating at the subsonic region for stars with mass loss rates of the order of $10^{-10} M_{\odot} \mathrm{yr}^{-1}$.

If the central object is a late type star, the subsonic heating could be also attributed to the dissipation of acoustic waves or Alfven waves, generated in the convection zone of the star. These waves can be selectively amplified or damped by radiative processes (Hearn 1972, 1973; MacGregor et al. 1979; Ibáñez 1985). The mechanical flux due to acoustic waves of rms velocity amplitude $\sqrt{\left\langle u^{2}\right\rangle}$ is $F_{\text {ac }}=1 / 2 \rho<u^{2}>c$ with $c$ the local speed of sound. For a photospheric heating flux of $F_{0} \simeq 0.27 \times 10^{3} \mathrm{erg} \mathrm{cm} \mathrm{cm}^{-2} \mathrm{~s}^{-1}$, acoustic waves with $\mathrm{rms}$ velocity fluctuations of the order of $\langle u\rangle \simeq 1 / 2 c$ could provide the heat flux. Moreover, the heating flux damping length, $l_{\mathrm{F}}$, shown in Fig. $4 \mathrm{~d}$ is consistent with acoustic wave damping. According to McWhirter et al. (1975) and for the density and temperatures considered here, the damping length is consistent with the dissipation of acoustic waves with periods shorter than $10^{3} \mathrm{~s}$, which is within the typical cut-off period which is estimated to be of the order of $\sim 3 \times 10^{6} \mathrm{~s}$ (Ulmschneider 1988). These results are also consistent with Ibáñez's calculations for sound wave amplification or damping in an externally heated fluid. In conclusion, both the heat flux and the heat damping length of the inner subsonic region could arise from acoustic wave dissipation.

\subsubsection{The supersonic, cooling envelope}

As a measure of radiative losses the black-body energy density radiation $q_{\mathrm{bb}}$ in the envelope regions where the fluid can be considered optically thick can be taken:

$q_{\mathrm{bb}}=\rho \boldsymbol{V} \cdot \nabla\left(\frac{\alpha_{\mathrm{SB}} T^{4}}{\rho}\right)$,

where $\alpha_{\mathrm{SB}}=7.56 \times 10^{-15} \mathrm{erg} \mathrm{cm}^{-3} \mathrm{deg}^{-4}$ and is the Stefan-Boltzmann constant. Because the actual thermal emission is less than that of a black-body, the estimated $q_{\mathrm{bb}}$ provides an upper bound for the cooling rate, as shown in Fig. 4b. In fact, the cooling rate $q$ is orders of magnitude smaller than $q_{\mathrm{bb}}$. This indicates that the supersonic region should be optically thin.

We can obtain agreement with the cooling rates of optically thin plasmas obtained by Schmutzler \& Tscharnuter (1993) even if we assume a mass loss rate of the order of $\dot{M}=10^{-8} M_{\odot} /$ yr. For comparison with the previous work, the cooling curve as well as the cooling rates $\sigma$ and $\Lambda=q n^{-2}$ are shown in Fig. 5. These loss rates estimates are also in agreement with the calculations of Neufeld \& Kaufman (1993) for emission in warm molecular gases and of Woitke et al. (1996) for shocked circumstellar envelopes of pulsating stars. We conclude that the cooling is radiative and in accordance with radiative cooling rates of optically thin media.

\subsection{The energetics of steady shell thermo-radiative case}

In order to describe the radiative acceleration that produces low terminal velocity winds from supergiants, we adopt the optically-thin-line radiative force given by Chen \& Marlborough's (1994) approximation, (also Vlahakis \& Tsinganos 1999; Kakouris \& Moussas 1998, 1997; Lamers 1986)

$\boldsymbol{F}_{\mathrm{rad}}=\rho \frac{k \Gamma \nu^{2} V_{\mathrm{p}}^{2}}{2 R_{*}}\left(\frac{R_{*}}{r}\right)^{2-\varepsilon}$,

with $\nu=V_{\text {esc }} / V_{\mathrm{p}}$ and $V_{\text {esc }}$ the escape velocity at the stellar surface. The force parameters $(k, \varepsilon)$ will be considered constants in this work.

The mechanism described in this paragraph applies both to early and late type supergiants. For application we use the prototype of LBVs, $P$ Cyg, as the central star following Paper I. The envelope of this star may possess a deceleration region (Kuan \& Kuhi 1975; Nugis et al. 1979) and also exhibits shells or blobs (Vakili et al. 1997). These outflows may not arise as thick-line-driven winds but from the absorption of a large number of the weak, optically thin spectral UV lines in the Balmer continuum (Lamers 1986). P Cyg also exhibits emission lines, and there is observational evidence of its cooling from the radio 

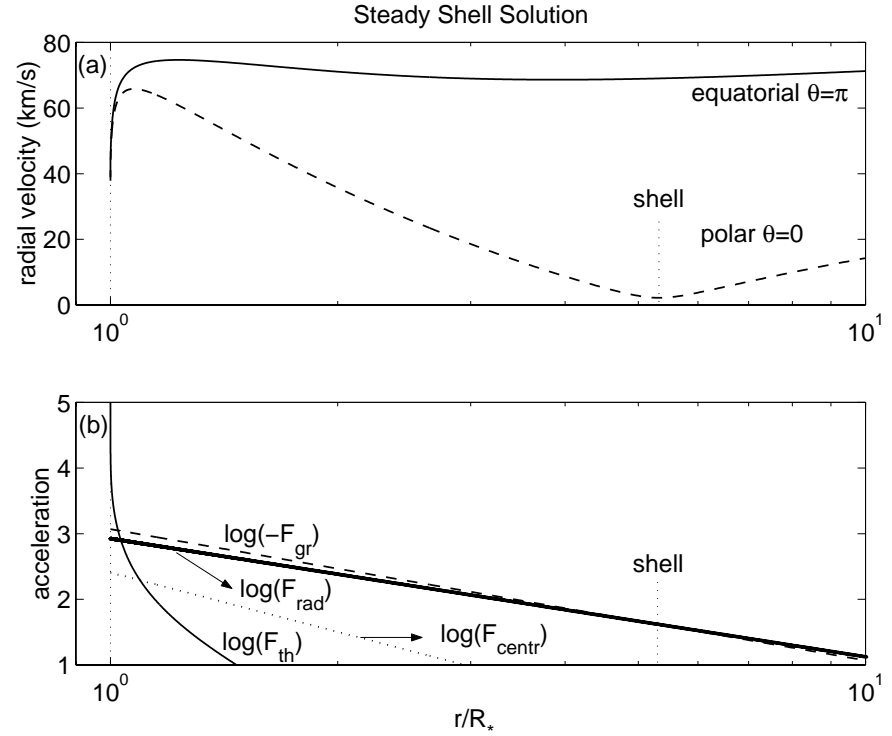

Fig. 6. The same with Fig. 1 for the thermo-radiative shell case. a) Radial velocities b) Force balance. The thermal acceleration curve $\boldsymbol{F}_{\text {th }}$ is polar and $\boldsymbol{F}_{\text {gr }}$ is the effective gravitational acceleration. Accelerations are in $V_{\mathrm{p}}^{2} / R_{*} \simeq 3 \times 10^{-7} \mathrm{~km} / \mathrm{s}^{2}$ units. The parameters of the solution are presented in Sect. 3.2.

emission from an enveloping clumpy nebula (Skinner et al. 1998). Parameters of the star are discussed extensively by Israelian \& de Groot (1999) and model parameters are the same with Paper I $\left(A=385, T_{\mathrm{p}}=10^{3} \mathrm{~K}, \lambda=50, k=\right.$ $0.25, \varepsilon=0.2)$. The mass loss rate is $1.5 \times 10^{-5} M_{\odot} \mathrm{yr}^{-1}$. The shell is formed at $\simeq 5.5 R_{*}$ with a shell mass of $1.45 \times 10^{-5} M_{\odot}$. The shell formation timescale is about $1 \mathrm{yr}$.

In early type stars there is no observational evidence for high temperatures, i.e. of the order of $10^{6} \mathrm{~K}$. However, the temperature may exceed the effective one by a factor of $\sim 5$ in its possibly existing chromosphere (Cidale 1998) or corona-like layer (Underhill \& Doazan 1982). The effective temperature of $P$ Cygni is $18200 \mathrm{~K}$, so the temperature is kept below $10^{5} \mathrm{~K}$ in this example (Fig. 8). The flow velocity (equatorial and polar) and force balance are presented in Fig. 6 . The radial velocities are less than the local escape speed at all distances. The thermal force dominates close to the stellar surface, decaying rapidly with distance and the radiative force balances the effective gravity at the shell radius. The inner envelope $\left(r<3 R_{*}\right)$ is similar to the non-radiative case. Polar regions, where the shell forms, exhibit three zones, i.e. an accelerating-decelerating-accelerating outflow. The effective gravitational force dominates in the intermediate decelerating region. The respective heating/cooling rates are shown in Fig. 7 and the effective polytropic index, $\alpha$, in Fig. 8. Equatorial $q$ or $\sigma$ as well as $\alpha$ are similar to the non-radiative case (Figs. 2, 3).

The subsonic region is similar to the non-radiative case, presented in the previous section. Heat conduction is negligible, $q_{\mathrm{c}}<10^{-7} q$, and the effective heat luminosity is $\mathcal{L}_{\mathrm{h}} \simeq 6.7 \times 10^{-7} L_{*}$. However, a turbulent conductivity

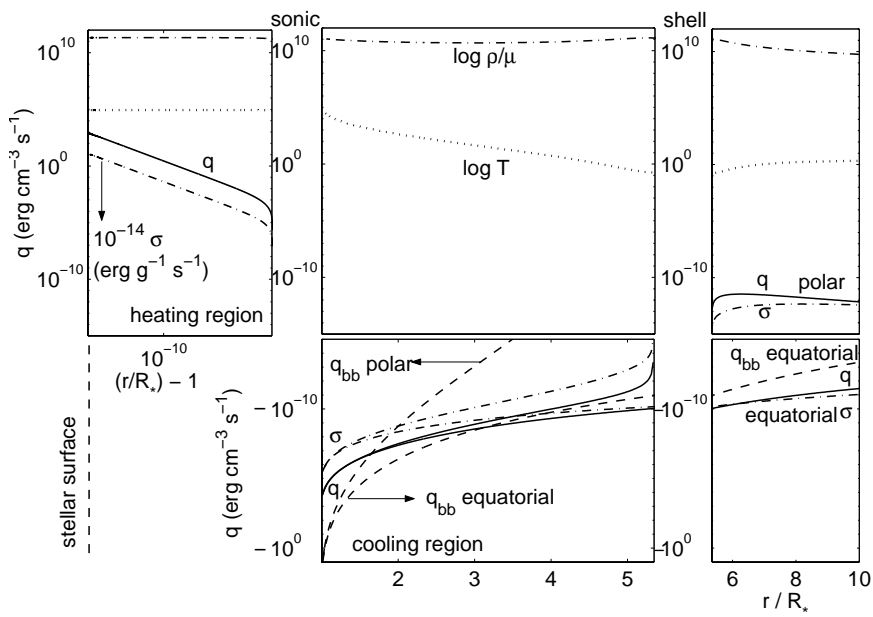

Fig. 7. The same with Fig. 2 for the thermo-radiative shell case. The polar temperature (dotted curve) and the polar number density (dash-dotted curve) are also shown. Polar and equatorial $\sigma$ (or $q$ ) are equal for $r<1.5 R_{*}$. For $r>1.5 R_{*}$ the equatorial $\sigma$ (or $q$ ) is similar to the non-radiative case (Fig. 2) while the polar values become zero at the shell distance and imply heating in the outer acceleration region. So, the three zone stellar envelope exhibits a heating-cooling-heating expansion. Note that $\sigma$ (dash-dotted curves) is multiplied by $10^{-14}$ everywhere to fit in scale and that the local black-body emission curves $q_{\mathrm{bb}}$ intersect the equatorial and polar $q$ at 3 and $1.5 R_{*}$ respectively due to the low temperature in the region in front of the shell.

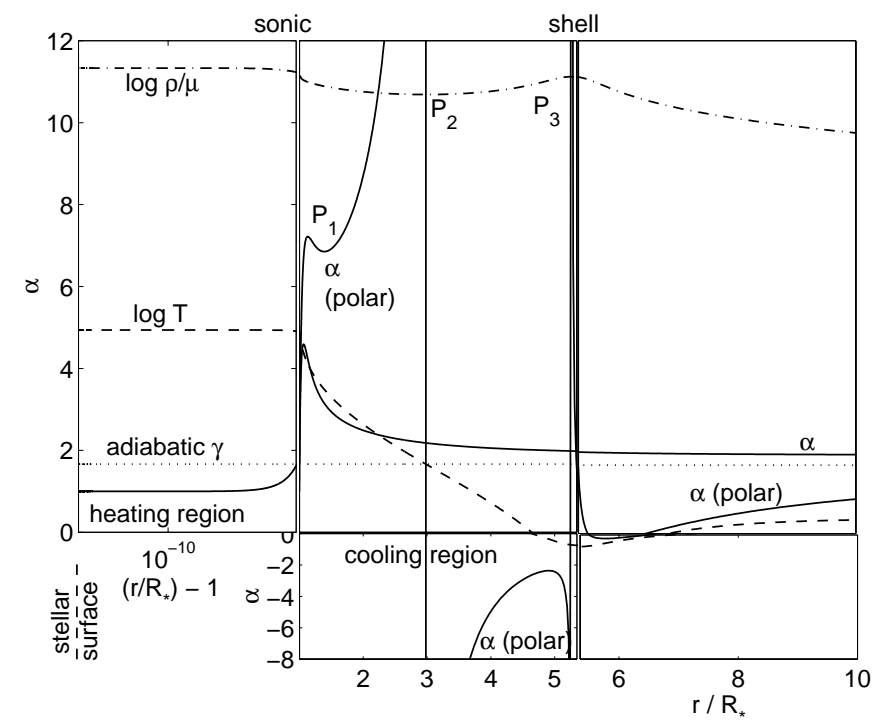

Fig. 8. The same with Fig. 3 for the thermo-radiative shell case. Equatorial effective polytropic index is similar to the nonradiative case (Fig. 3) while the polar one increases at $r \simeq$ $1.5 R_{*}$ and becomes negative for $3 R_{*} \leq r \leq 5.5 R_{*}$.

can provide the required heat transfer given that photospheric eddy rms velocities of $\sim 10-20 \mathrm{~km} \mathrm{~s}^{-1}$ have been observed (Underhill \& Doazan 1982). The heat damping length is $l_{\mathrm{q}} \simeq 10^{3} \mathrm{~km}$ very close to the photosphere and $2 \times 10^{4} \mathrm{~km}$ in the subsonic region. This is consistent both with a conductive fall-off as well as with the damping 

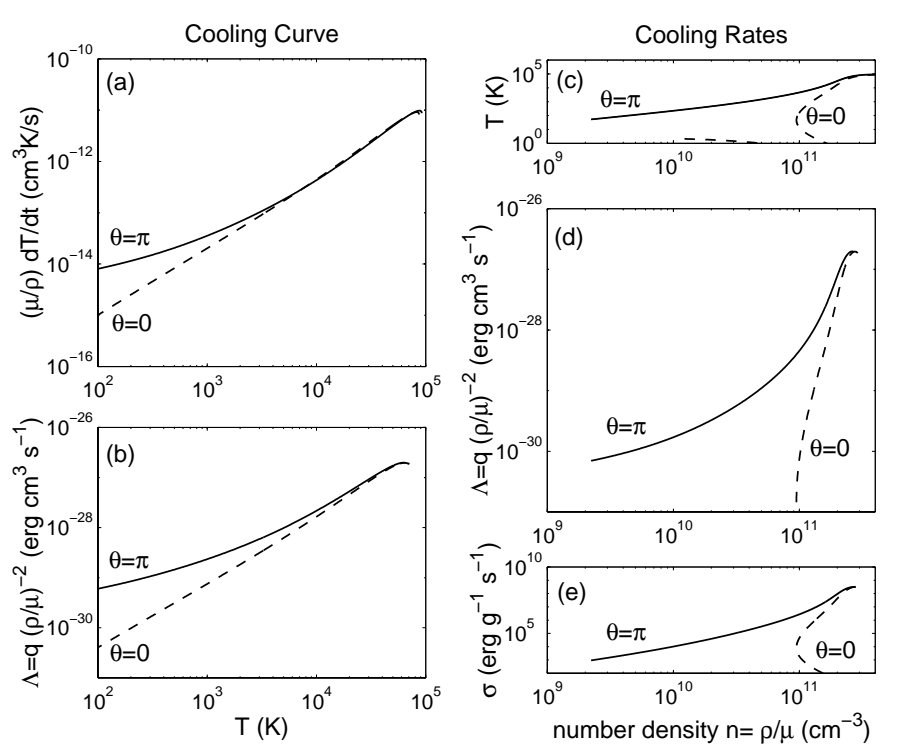

Fig. 9. The same with Fig. 5 for the thermo-radiative shell case. The solid lines are equatorial and the dashed are polar.

associated with acoustic wave dissipation of waves with periods shorter than $50 \mathrm{~s}$ (McWhirter et al. 1975). The photospheric heat flux $F_{0} \simeq 5 \times 10^{6} \mathrm{erg} \mathrm{cm}^{-2} \mathrm{~s}^{-1}$, requires that the acoustic waves have rms amplitudes of the order of $\sqrt{\left\langle u^{2}\right\rangle} \simeq 0.35 \mathrm{c}$. For early type supergiants, acoustic waves arise from the pulsations of the star. We conclude that the required subsonic heating can be explained by the combination of turbulent conduction and acoustic wave dissipation, as well as by radiative absorption due the large number of weak spectral lines in UV where $P C y g$ emits most of its luminosity. An amount of heat, much less than the subsonic, is also needed in the post-shell region. This secondary heating cannot be attributed to wave damping and it will be discussed in a next paragraph.

The cooling curve and cooling rates for envelope regions of $T>100 \mathrm{~K}$ are shown in Fig. 9. The equatorial specific cooling rate $\sigma$ (Fig. 9e) is the same with Fig. 5e and in accordance with Woitke et al. (1996). Furthermore, the cooling curve (Fig. 9a) and cooling rate $\Lambda=q n^{-2}$ (Fig. 9b) are in accordance with the isometric or isobaric emission calculation of Schmutzler \& Tscharnuter (1993) for an optically thin plasma with negligible metallicity (see their Fig. 6, $Z<0.01$ ), as expected in early type stars. The equatorial $\Lambda$ (Fig. 9d) is in accordance with molecular cooling (Neufeld \& Kaufman 1993). The double valued polar curves in plots $9 \mathrm{e}, \mathrm{f}$, correspond to different cooling rates of equal density but different temperature gas, i.e. at the inner region $r<2 R_{*}$ (warm) and at shell location $r>3 R_{*}$ (cool). In addition, black-body emission $q_{\mathrm{bb}}$ (Fig. 7, dashed lines) exceed polar radiative cooling only for $r<2 R_{*}$. The polar effective polytropic index (Fig. 8) becomes negative for $r>3 R_{*}$, i.e. the local density minimum. Polar $\alpha$ tends asymptotically to 2 in the same manner as in equatorial plane. Since negative $\alpha$ or $\alpha<1$ makes little thermodynamic sense, the intermediate deceleration region needs further analysis.

The intermediate envelope (Fig. 8) is confined between the post-sonic point $P_{1}$ and the shell distance $P_{3}$. The local density is minimum at $P_{2}$. Between the sonic radius and $P_{1}$, continuum (thermal) emission is expected in optical and near-IR frequencies. The region between $P_{1}$ and $P_{2}$ exhibits large values of $\alpha$ and the local thermodynamics are almost isometric. The respective radiative cooling is in agreement with estimations obtained from the isometric calculations of Schmutzler \& Tscharnuter (1993). The continuum emission from this region is expected mainly in the infrared.

The region $P_{2}$ to $P_{3}$ is of special interest because the drop in temperature and pressure is accompanied by a density rise and radiative cooling (the opposite is valid for $\sim 1 R_{*}$ in the post-shell region). As a result, the effective polytropic index becomes negative, indicating that the local thermodynamic equilibrium may not be a good approximation. Although $\alpha<1$ values are not acceptable in the present work and they are treated as indication that the medium is convectively unstable (e.g. Shu 1974; Ryu \& Goodman 1992) we find that this is not the case in the region $P_{1}-P_{2}$. Since the radial velocity is small (less than $20 \mathrm{~km} \mathrm{~s}^{-1}$ ), the shell configuration can be considered quasi-static and the calculation of the adiabatic lapse rate (Landau \& Lifshitz 1959) is possible in order to approximately identify convective instability. The temperature distribution is convectively stable when

$N=\frac{\mathrm{d} T}{\mathrm{~d} r}+\frac{a_{\mathrm{tot}}}{c_{\mathrm{p}}}>0$,

where $a_{\text {tot }}$ is the total acceleration on a fluid element. $N$ was found positive in the supersonic envelope of the non-radiative example, presented in the previous section. The same holds for the shell region as well as for the rest stellar envelope presented in this section. As a result, the temperature profile can be considered convectively stable. Only the vicinity of the shell is a candidate region for convective instability. If convective motions are possible, the situation will be similar to the negative polytropic index regions found from spacecraft measurements at the terrestrial magnetopause (Pudovkin et al. 1997). We have to note that local turbulent motions are in agreement with spectroscopic shell observations (line profiles) and they are also supported by observations of the clumpy radio nebula around $P$ Cyg with fast variability (Skinner et al. 1998). Since thermal motions correspond to the temperature gradient, corrections of the latter due to diffuse radiation are useful (Millar \& Marlborough 1999). The required heating in the post-shell region is correlated with local thermal conduction and radiative absorption.

A qualitative view of the different polar regimes for the envelope of $P C y g$ is shown in Fig. 10. The right plot shows the number density, the central is a logarithmic density contour plot illustrating the shell and the left summarizes qualitatively the discussion of the present paragraph for the different envelope heating/cooling regions. 


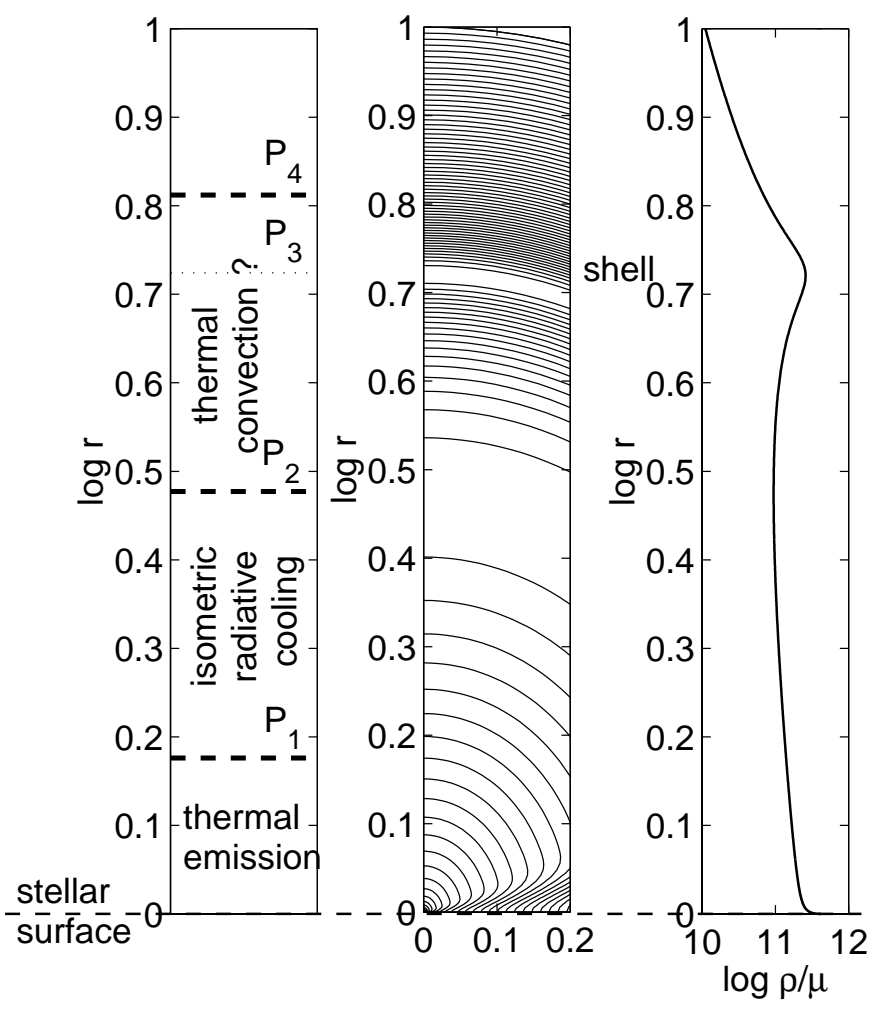

Fig. 10. A view of the shell along the vertical (rotational) axis of the central body. The plots show logarithmic density contours (central), the number density radial dependence (right) and the different domains of the envelope (left) according to the discussion of Sect. 3.2.

\section{Conclusions}

The energy sources required for energy balance in a circumstellar envelope described by Kakouris \& Moussas (1997) were calculated. In the non-radiative case of a latetype supergiant $(\mathrm{K} 5 \mathrm{I})$ we found that the heating in the subsonic region and the radiative cooling in the supersonic depend on the mass loss rate and the respective rates are consistent with heat conduction, acoustic wave dissipation and effective emission by the expanding envelope gas. The analysis shows that our model is essentially a "coronal wind" model with small laminar thermal conduction and effective heat luminosity 8 orders of magnitude less than the stellar. Assuming LTE, the implied effective polytropic index indicates that the subsonic envelope is isothermal. The index is found to be less than 4 in the supersonic (cooling) envelope and at large distances it approaches 2 . The effective polytropic index is reasonable, i.e. close to the usual values $([1, \gamma=5 / 3])$ of astrophysical gases in thermal equilibrium, despite the lack of initial assumptions on thermodynamics.

The energy balance analysis was repeated for the case of stellar atmospheres with extensive polar shells or blobs, adding the appropriate thin-line radiative forcing. For this investigation, $P$ Cygni was chosen as the central star. The application was limited to the analytic, self-consistent hydrodynamic description of dense blobs or shells in stellar envelopes. The subsonic and the equatorial regions of the envelope were found to involve similar dynamics with the non-radiative case. The analysis implies that the physical processes in polar regions, where the steady shell forms, are different. There, the stellar envelope is separated in three zones, the two outer accelerating and the intermediate decelerating. In the decelerating region the fluid radiatively cools and is therefore the candidate location for emission line formation. At larger distances from the star a cool dense shell is formed, characterized by a reduced expansion and possible convective motions. This is consistent with early type star's observations but a detailed stability analysis is needed to determine the convective stability of this region. The current results are in agreement with the early work of Nugis et al. (1979).

We remind the reader that the shell is formed under the assumption that the thin radiative force for weak spectral lines absorption is of the order of the effective gravity. Such shells sensitively depend on the assumed parameters like optical depth, number of optically thin lines, local ionization conditions, stellar luminosity etc. For a more realistic investigation, we must take into account the line absorption coefficient at several distances from the star as well as the long term variability of the force parameters. This variability does not affect the present description if it occurs over a much longer period than the shell formation timescale $\left(M_{\text {shell }} / \dot{M}\right)$. Any destruction of the steady state equilibrium will cause the shell in motion to tend towards larger radii where the density is lower. The time needed for the appearance of another shell under the same mechanism depends on the mass loss rate and is about $1 \mathrm{yr}$ for P Cyg.

Finally, it has to be noted that stationary shells may also form at the equatorial regions of stars, if we adopt similar 2-D, non-polytropic models with maximum mass flux density in the stellar equator, as in the model of Lima \& Priest (1993). This case will be considered in a forthcoming paper.

Acknowledgements. The author acknowledges the Greek National Scholarships Foundation (NSF) for his postdoctoral research funds and Professors Xenophon Moussas and Petros Ioannou for stimulating discussions and helpful comments.

\section{References}

Cally, P. S. 1990, ApJ, 355, 693

Castor, J. I., Abbott, D. C., \& Klein, R. I. 1975, ApJ, 195, 157

Chen, H., \& Marlborough, J. M. 1994, ApJ, 427, 1005

Cidale, L. S. 1998, ApJ, 502, 824

Eddington, A. S. Sir 1938, MNRAS, 99, 4

Hauck, B., \& Jaschek, C. 2000, A\&A, 354, 157

Hearn, A. G. 1972, A\&A, 19, 417

Hearn, A. G. 1973, A\&A, 23, 97

Ibáñez, S. M. H. 1985, ApJ, 290, 33

Israelian, G., \& de Groot, M. 1999, Space Sci. Rev., 90, 493

Jura, M. 1984, ApJ, 282, 200

Kakouris, A. 1997, Ph.D. Thesis, University of Athens

Kakouris, A., \& Moussas, X. 1996, A\&A, 306, 537

Kakouris, A., \& Moussas, X. 1997, A\&A, 324, 1071

Kakouris, A., \& Moussas, X. 1998, A\&A, 333, 678 (Paper I) 
Kuan, P., \& Kuhi, L. V. 1975, ApJ, 199, 148

Lamers, H. J. G. L. M. 1986, A\&A, 159, 90

Lamers, H. J. G. L. M, \& Cassinelli, J. P. 1999, Introduction to Stellar Winds (Cambridge University Press)

Landau, L. D., \& Lifshitz, E. M. 1959, Fluid Mechanics (Pergamon Press, Bristol), 8

Lima, J. J. G., \& Priest, E. R. 1993, A\&A, 268, 641

Low, B. C., \& Tsinganos, K. 1986, ApJ, 302, 163

MacGregor, K. B., Hartmann, L., \& Raymond, J. C. 1979, ApJ, 231, 514

McWhirter, R. W. P., Thonemann, P. C., \& Wilson, R. 1975, A\&A, 40, 63

Mauron, N., \& Huggins, P. J. 2000, A\&A, 359, 707

Millar, C. E., \& Marlborough, J. M. 1999, ApJ, 516, 276

Neufeld, D. A., \& Kaufman, M. J. 1993, ApJ, 418, 263

Nugis, T., Kolka, I., \& Luud, L. 1979, Mass loss and evolution of O-type stars (Reidel Dordrecht), 39

Olofsson, H., Bergman, P., Lucas, R., et al. A\&A, 330, L1

Pudovkin, M. I., Meister, C. V., Besser, B. P., \& Biernat, H. K. 1997, JGR, 102, A12, 27,145

Ryu, D., \& Goodman, J. 1992, ApJ, 388, 438

Sauty, C., \& Tsinganos, K. 1994, A\&A, 287, 893

Schmutzler, T., \& Tscharnuter, W. M. 1993, A\&A, 273, 318
Shu, F. H. 1974, A\&A, 33, 55

Skinner, C. J., Becker, R. H., White, R. L., et al. 1998, MNRAS, 296, 669

Totten, T. L., Freeman, J. W., \& Arya, S. 1995, JGR, 100, A1, 13

Totten, T. L., Freeman, J. W., \& Arya, S. 1996, JGR, 101, A7, 15,629

Ulmschneider, P. 1970, A\&A, 4, 144

Ulmschneider, P. 1977, A\&A, 60, 413

Ulmschneider, P. 1988, A\&A, 197, 223

Underhill, A., \& Doazan, V. 1982, B stars with and without emission lines, NASA SP-456, 136

Vakili, F., Mourard, D., Bonneau, D., Morand, F., \& Stee, P. 1997, A\&A, 323, 183

Viala, Y. P., \& Horedt, G. 1974, A\&A, 33, 195

Villada, M., Rossi, C., Polcaro, V. F., \& Giovannelli, F., 1999, A\&A, 344, 277

Vlahakis, N., \& Tsinganos, K. 1999, MNRAS, 307, 279

Winters, J. M., Keady, J. J., Gauger, A., \& Sada, P. V. 2000, A\&A, 359, 651

Woitke, P., Krüger, D., \& Sedlmayr, E. 1996, A\&A, 311, 927

Zuckerman, B. 1980, ARA\&A, 18, 263 